\section{Insights on the operation of Me-S batteries: a modeling perspective}

Timo Danner ${ }^{* 1,2}$, Raphael Richter 1,2 , Joachim Häcker ${ }^{1}$, Brigitta Sievert ${ }^{1}$, Norbert Wagner ${ }^{1}$, K. Andreas Friedrich ${ }^{1}$, Arnulf Latz ${ }^{1,2,3}$

${ }^{1}$ Institute of Engineering Thermodynamics, German Aerospace Center (DLR), Stuttgart, Germany; ${ }^{2}$ Helmholtz Institute Ulm for Electrochemical Energy Storage (HIU) and ${ }^{3}$ Institute of Electrochemistry, University of Ulm, Ulm, Germany

\section{Motivation}

Development, understanding \& rational design of Metal-Sulfur batteries Mg-Sulfur batteries:

+High volumetric energy density - Low rate performance

+Cheap \& abundant materials -Short cycle life

$\rightarrow$ Transfer of knowledge \& methodologies from Li-Sulfur

\section{Cell model [1]}

\section{- Electrolyte}

Mass conservation:

$\frac{\partial \varepsilon_{\mathrm{elyte}} c_{i}}{\partial t}=-\nabla N_{i}+\sum_{m} a_{m}^{v} v_{i, m} r_{i, m} 0=\sum_{i} z_{i} F\left(-\nabla N_{i}+\sum_{m} a_{m}^{v} v_{i, m} r_{i, m}\right)$

Species flux by Nernst-Planck equation (Diffusion + Migration):

$N_{i}=-D_{i} \nabla c_{i}-z_{i} \frac{F}{R T} D_{i} c_{i} \nabla \phi_{\text {eylte }}$

- Solids (charge conservation)

$0=-\kappa_{\text {elode }}^{\text {eff }} \nabla \phi_{\text {elode }}-\sum_{m \in \text { CTR }} a_{m}^{v} n_{m} F r_{i, m}$

Reaction rate (Butler-Volmer-Type):

$r_{m}=k_{f}^{0} \frac{a_{\text {ed }}^{1-\alpha} a_{\text {prod }}^{\alpha}}{\gamma}\left(e^{-\frac{\alpha}{R T} \Delta \bar{\mu}_{m}}-e^{-\frac{\alpha}{R T} \Delta \bar{\mu}_{m}}\right)$

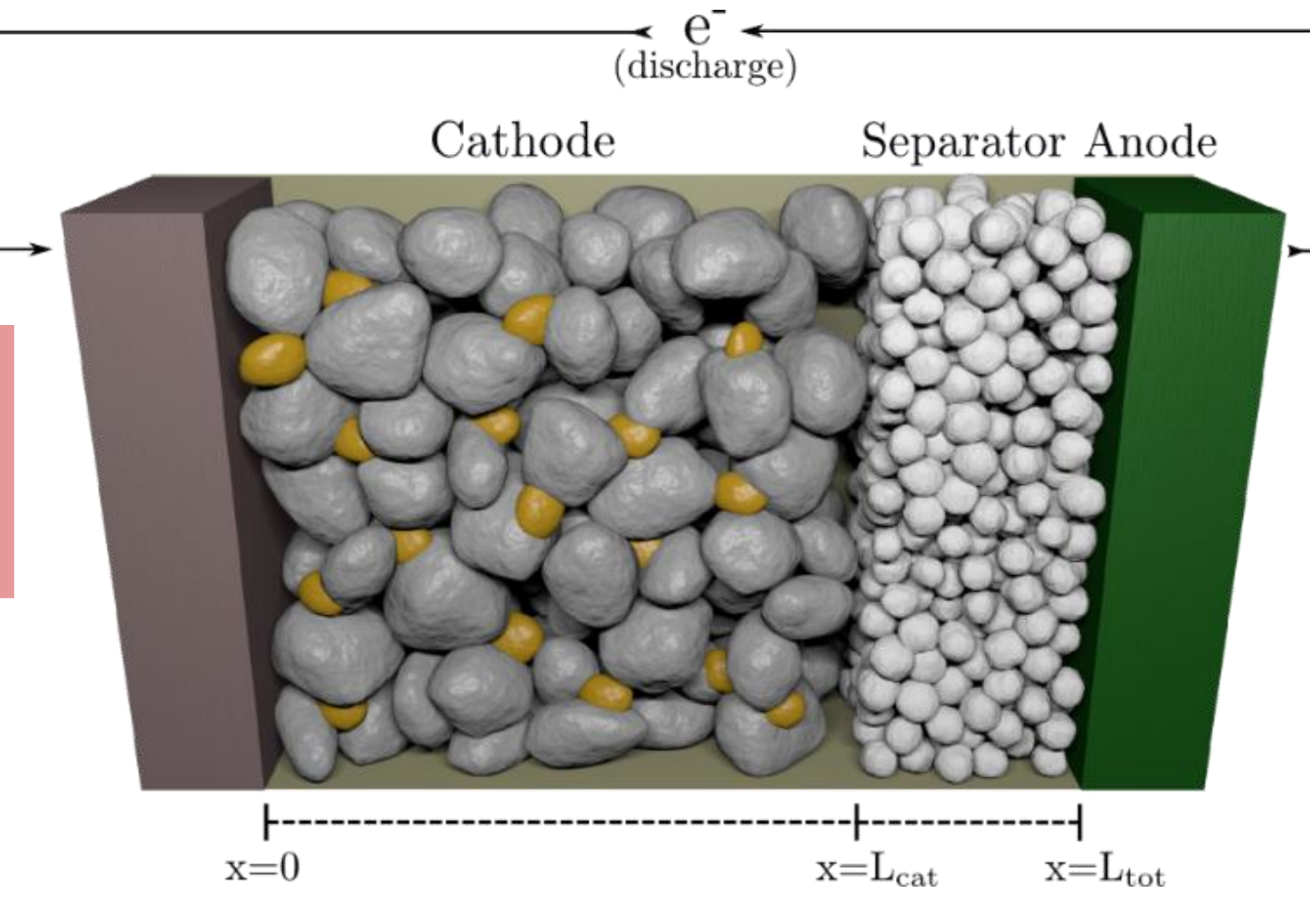

$\Delta \bar{\mu}_{m}=n F\left(\phi_{\text {elode }}-\phi_{\text {elyte }}-U_{\text {eq }}\right)$ where $U_{\text {eq }}=U_{\text {eq }}^{\ominus}+R T \ln \frac{a_{\text {prod }}}{a_{\text {ed }}}$

\section{Particle model [2]}

$\rightarrow$ The whole cell model in a spherical particle

- Coupling to cell model

- Electrolyte: Mass \& charge conservation

$\left.\dot{N}_{i}\right|_{r=R_{\mathrm{p}}}=-D_{i}\left(\left.\frac{\partial c_{i}}{\partial r}\right|_{r=R_{\text {p }}}+\left.z_{i} \frac{F}{R T} D_{i} c_{i} \frac{\partial \phi_{e}}{\partial r}\right|_{r=R_{\mathrm{p}}}\right.$

- Carbon matrix: Same potential

$\left.i_{F}^{\operatorname{mac}}\right|_{r=R_{\mathrm{p}}}=\int \frac{4 \pi r^{2} i_{F}(r) d r}{4 \pi R_{\mathrm{p}}^{2}}$

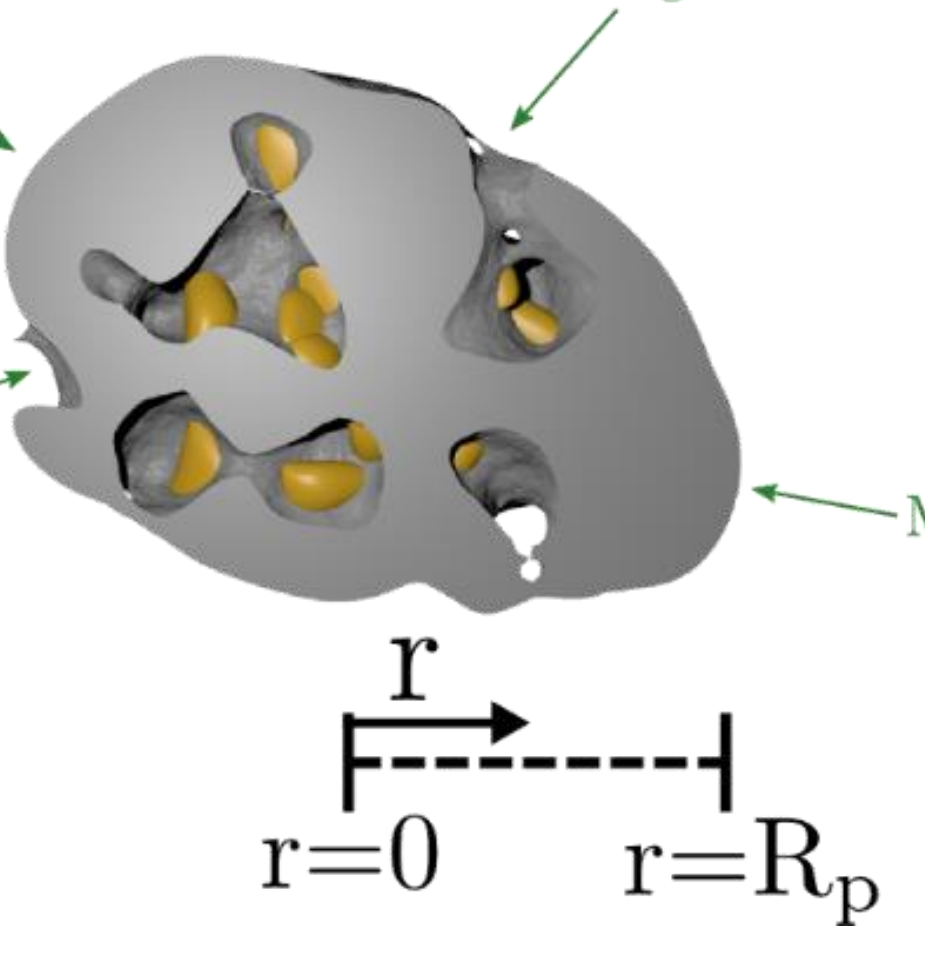

\section{Nucleation \& growth}

- Previous work [2]: Phenomenological treatment

$\begin{aligned} \frac{\partial \varepsilon_{k}}{\partial t}=\frac{\mathrm{MW}_{\mathrm{k}}}{\rho_{\mathrm{k}}} \sum_{m} a_{m}^{v} v_{k, m} r_{m} & \rightarrow \text { Surface passivation: } a_{m}^{\mathrm{v}}=a_{m, 0}^{\mathrm{v}}\left(\frac{\varepsilon_{\mathrm{elyte}}}{\varepsilon_{0}}\right)^{1.5}\end{aligned}$ $r_{m}=k_{f}^{0} \frac{a_{\text {ed }}^{1-\alpha} a_{\text {prod }}^{\alpha}}{\gamma}\left(e^{-\frac{\alpha}{R T} \Delta \bar{\mu}_{m}}-e^{-\frac{\alpha}{R T} \Delta \bar{\mu}_{m}}\right)$ where $\Delta \bar{\mu}_{m}=R T \ln \left(\mathrm{K}_{\mathrm{sp}} \frac{\mathrm{a}_{\text {prod }}}{\mathrm{a}_{\mathrm{ed}}}\right)$

- Now: Classical theory of nucleation \& growth

Particle size distribution $N_{k}(r)=f_{k}(r) d r$ :

$\frac{\partial f_{k}(r)}{\partial t}=\frac{\dot{N}_{k}^{\text {nuc }}}{d r_{0}} \delta_{0}-\frac{\partial}{\partial r}\left(f_{k} \frac{\mathrm{d} r_{k}}{\mathrm{~d} t}\right)$

Nucleation... surface energy [3] $\Delta G=4 \pi r_{k}^{2} \gamma+\frac{4}{3} \pi r_{k}^{3} \Delta G_{v}$

$\dot{N}_{k}^{n u c}=N_{0} Z D_{i} a^{2} \exp \left(-\frac{\Delta G_{\text {crit }}}{k_{B} T}\right)$

$$
\frac{d r}{d t}=\frac{D v_{k}^{m} c_{i}}{r_{k}+\left(D / k^{0}\right)}\left(1-S_{i}\right)
$$

Volume fraction:

$\varepsilon_{k}=\int \frac{4}{3} \pi r^{3} f_{k}\left(r_{k}\right) d r_{k}$

$\rightarrow \varepsilon_{\text {elyte }}=1-\sum_{k} \varepsilon_{k}$

Surface area: $a_{k}^{v}=\int \pi r^{2} f_{k}\left(r_{k}\right) d r_{k}$

$$
\rightarrow a_{m}^{v}=a_{m}^{v, 0}-\sum_{k} a_{k}^{v}
$$

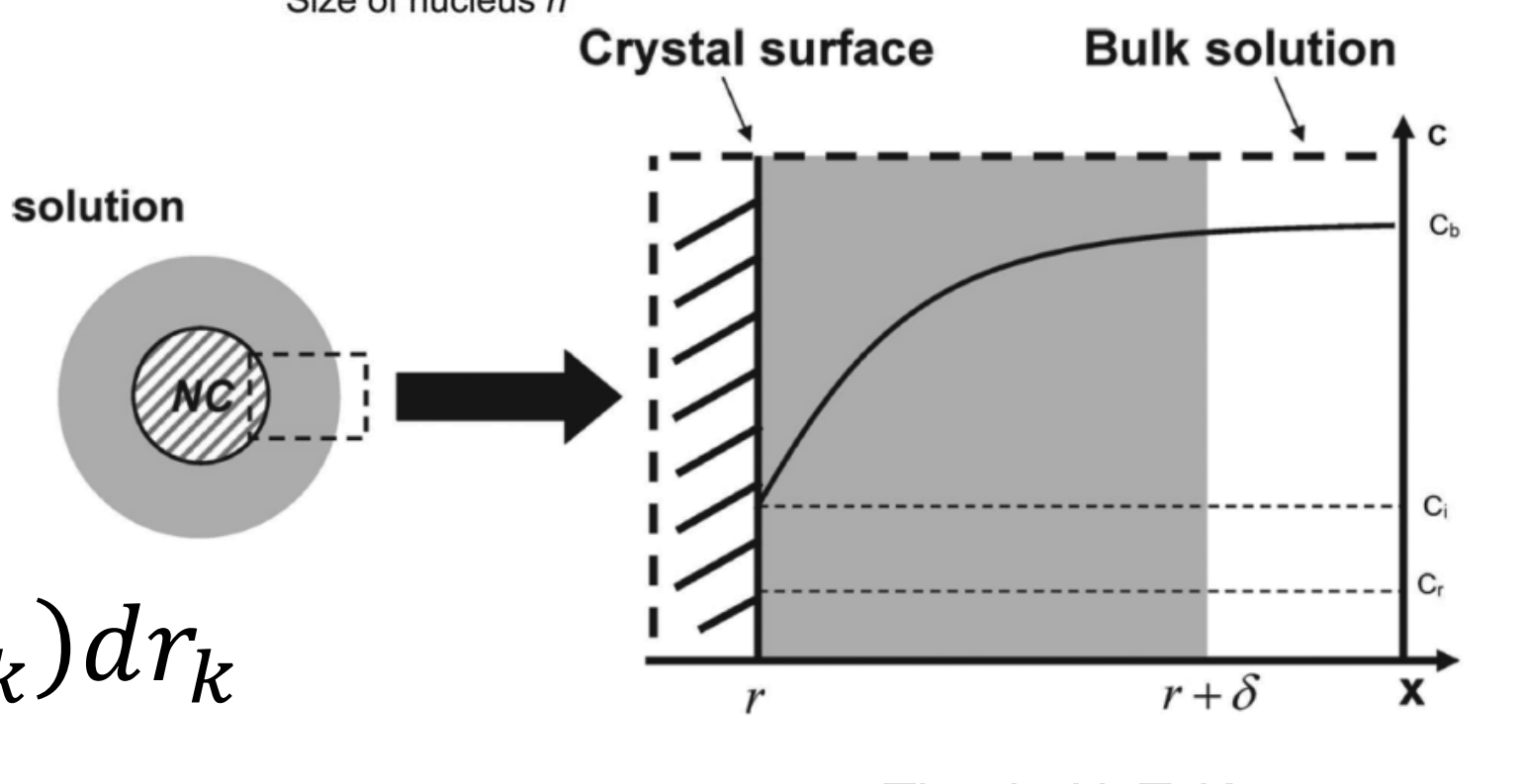

Thanh, N. T. . . et al
Chem. Rev. 114.

[1] A. F. Hofmann et al., J Power Sources 259, 300-310 (2014).

[3] H. Park et al., J Phys Chem C 119, 4675-4683 (2015).

\section{Li-S cell simulation}

- Discharge \& charge simulations

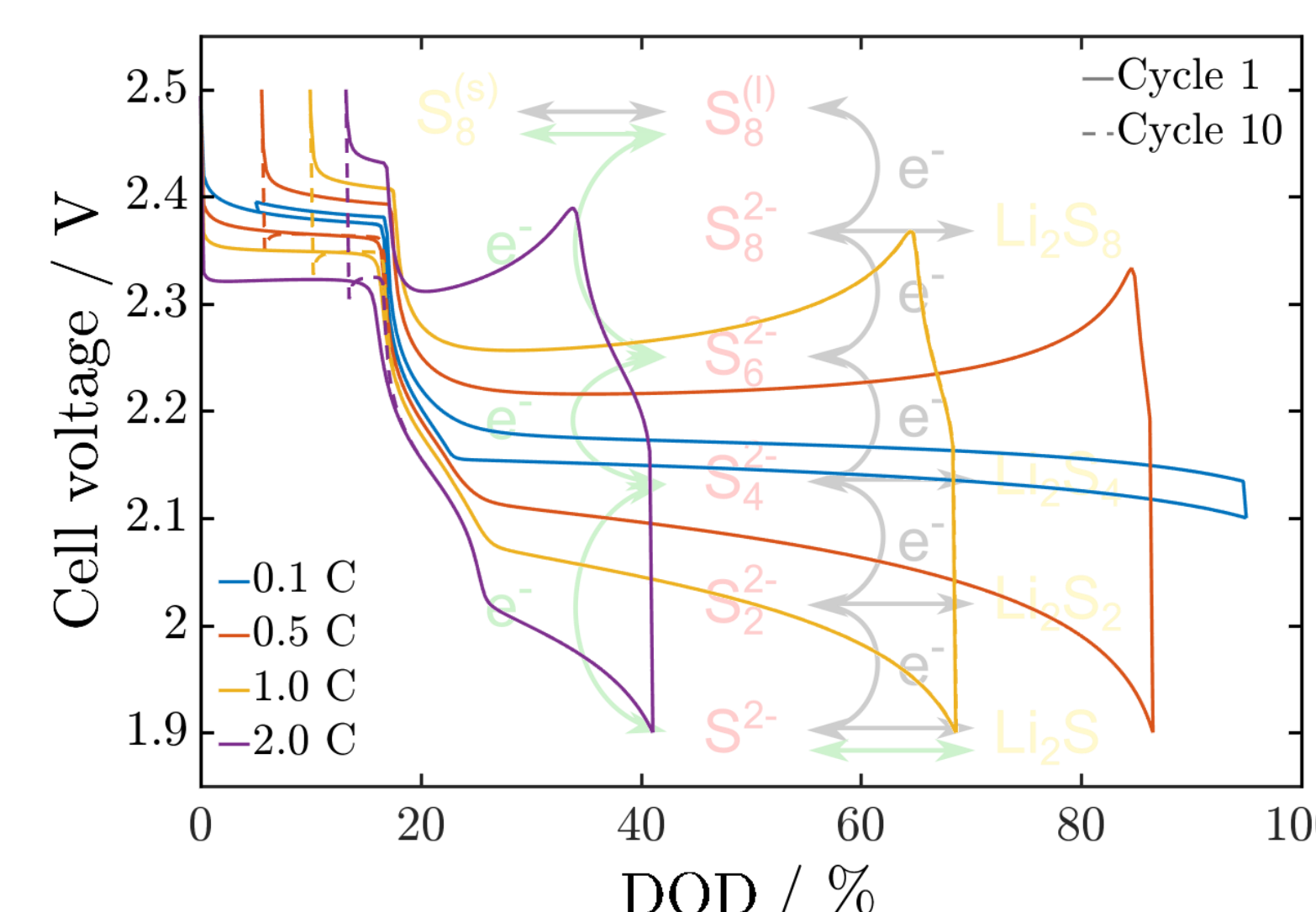

- Cycling data at high C-rates:

- Loss in capacity \&

voltage peak during charge

- Decreasing $\mathrm{Li}_{2} \mathrm{~S}$ crystal size

$\rightarrow$ Surface passivation
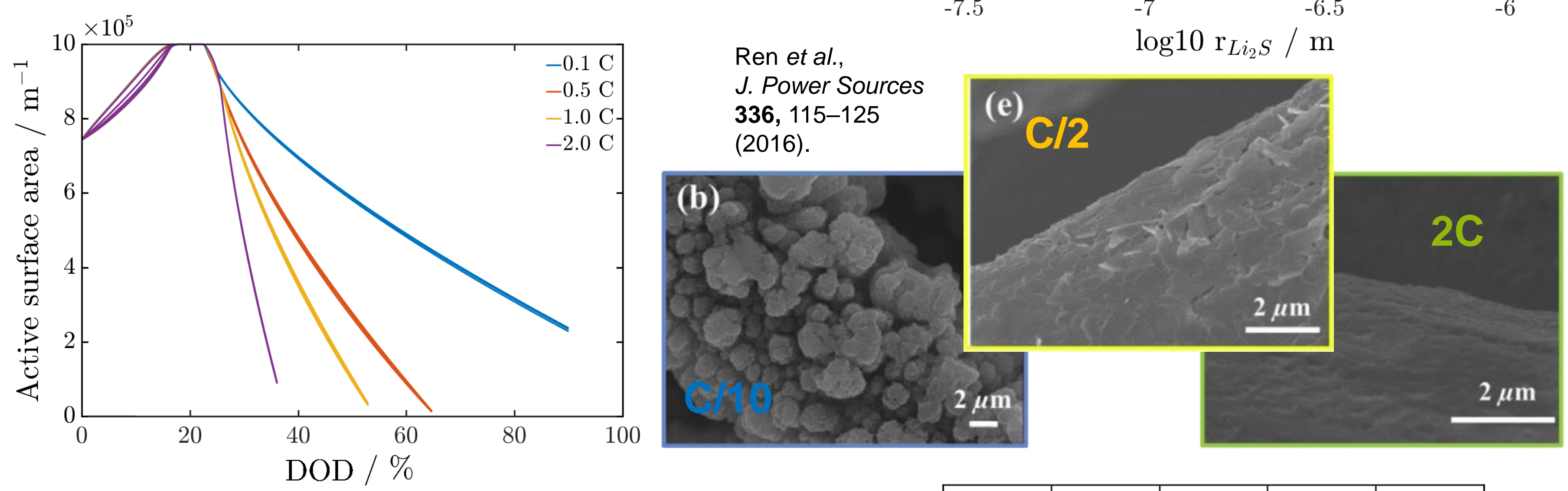

- Constant sulfur loading: 20 vol-\%

$\rightarrow$ Current collector: large particles

$\rightarrow$ Separator: Small particles

$\rightarrow$ Redistribution of sulfur

- Influence of polysulfide shuttle on sulfur redistribution?

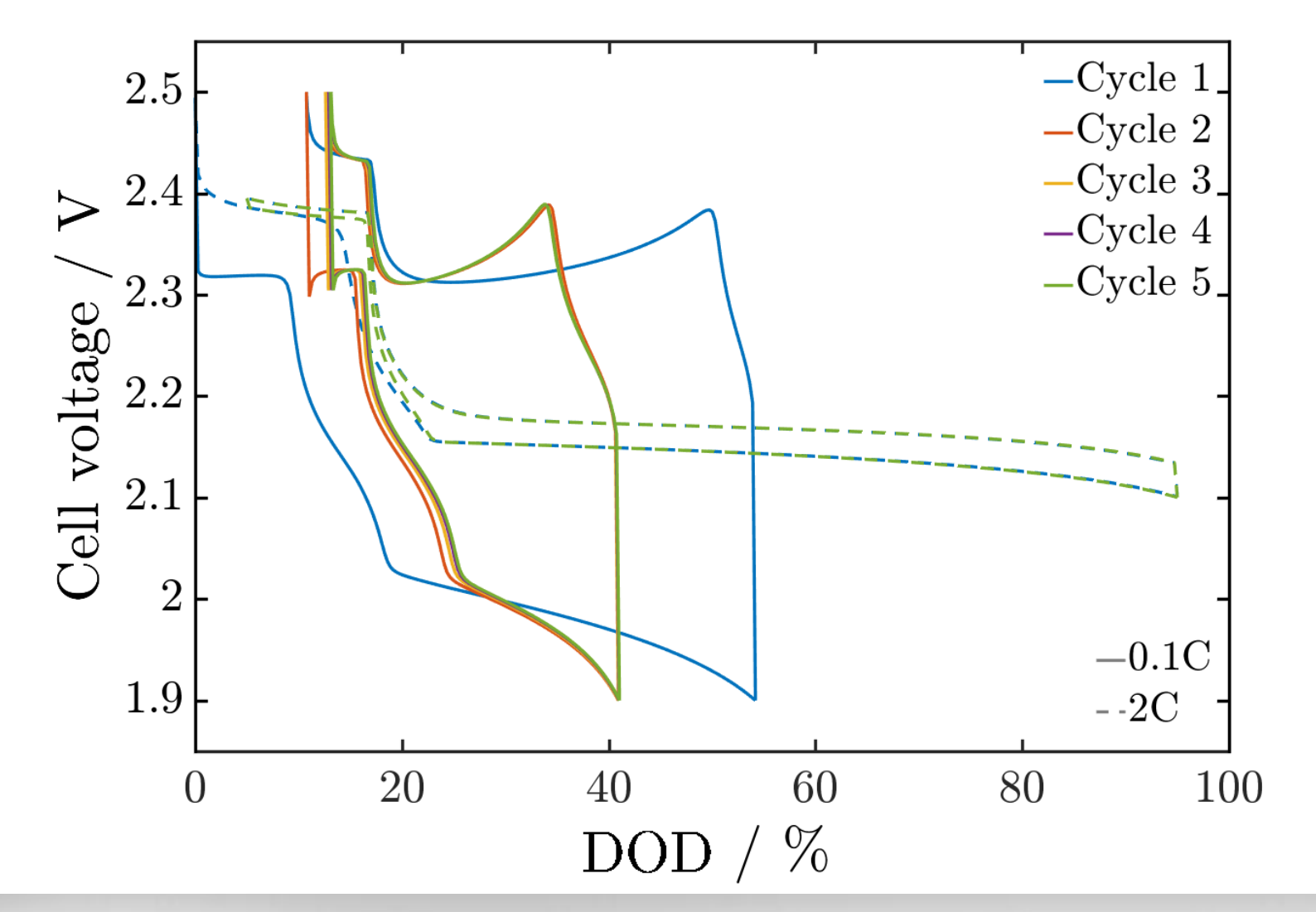

\section{Mg-S cell simulation}

- S/C composite cathode preparation Milled vs. Melt infiltration

$\rightarrow$ Cell model vs. Cell+particle model

- Mg foil anode

- Electrolyte: Mg(HMDS) $)_{2}$ / TEG:DEG [4] size distribution \& $\mathrm{no} \mathrm{Li}_{2} \mathrm{~S}$ $\rightarrow$ Dissolution of $\mathrm{S}_{8}$

$\rightarrow$ Growth of $\mathrm{Li}_{2} \mathrm{~S}$

$\rightarrow$ Reverse processes during charge $\rightarrow$ No degradation: Reversible cycling

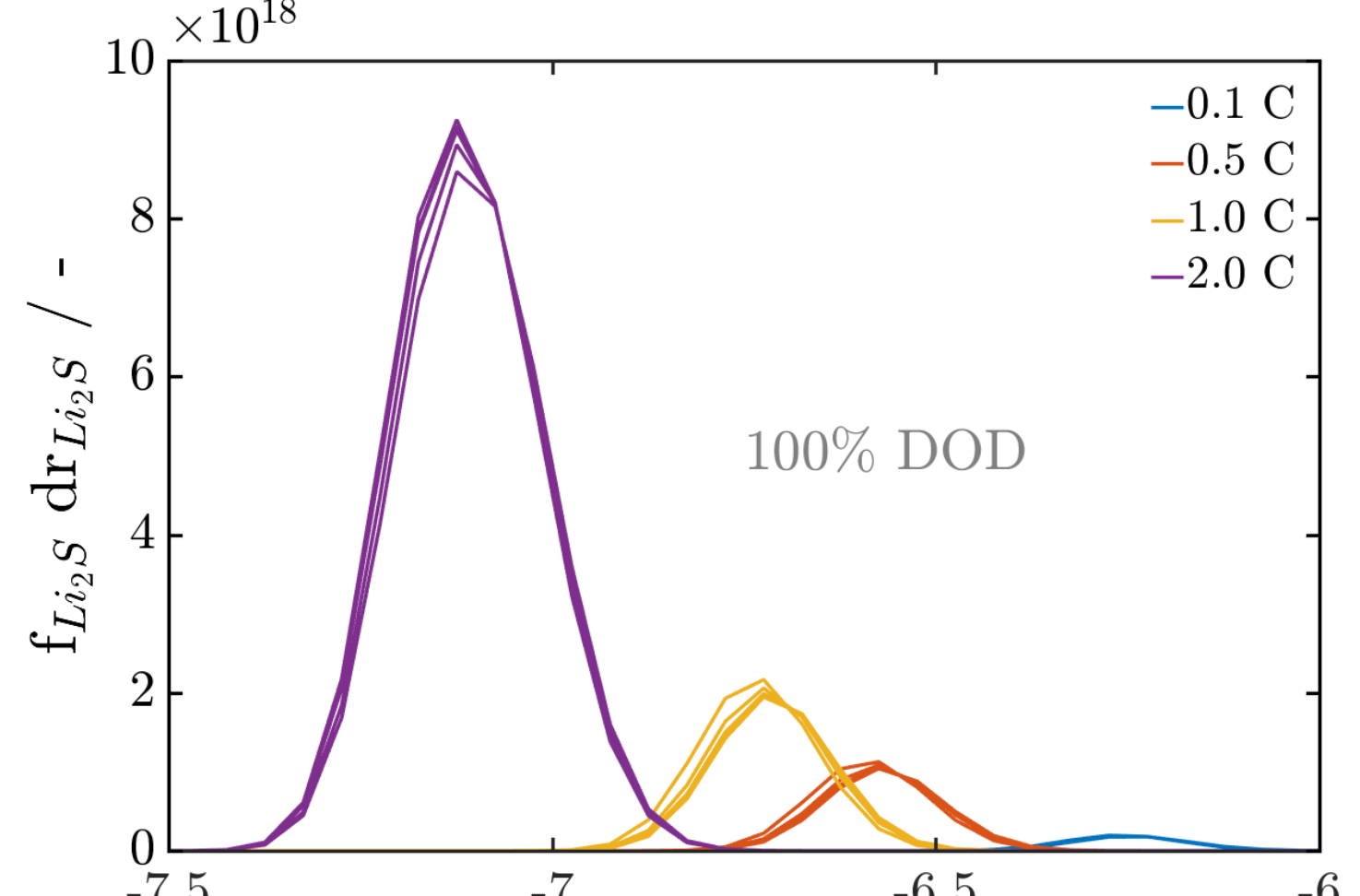
$\log 10 \mathrm{r}_{L i_{2} S} /$

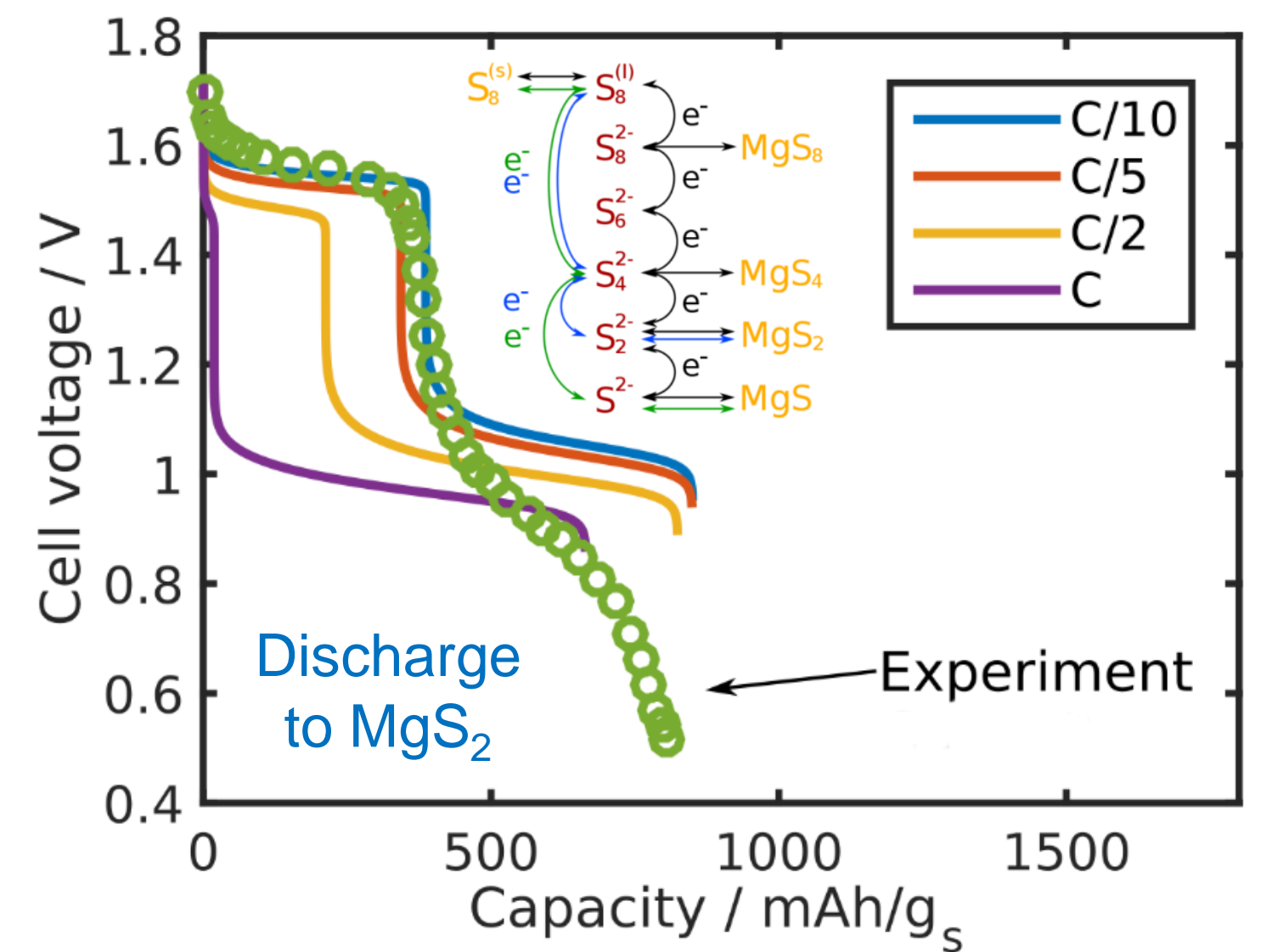

- Discharge mechanism?

$\rightarrow$ Capacity: Possible end product $\mathrm{MgS}_{2}$

- Sloping potential in $2^{\text {nd }}$ 'plateau'

- Surface passivation? ( $\rightarrow$ nucleation)

- Viscosity effects? ( $\rightarrow$ no nucleation)

$\rightarrow$ Experimental evidence needed!

- Infiltrated particles: Polysulfide 'bleeding' \& shuttle

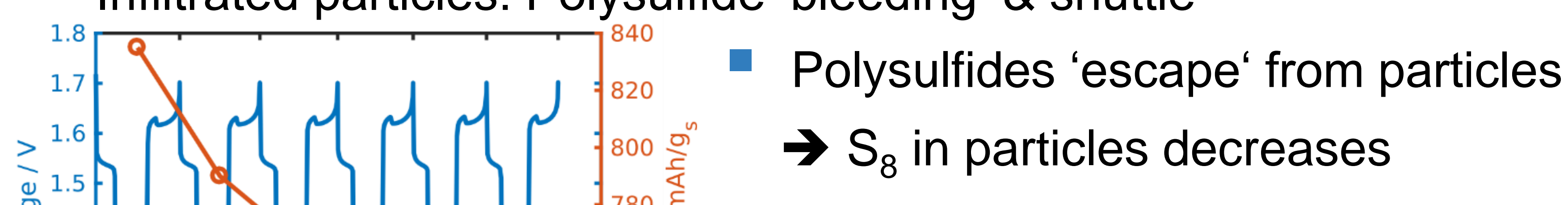

- Polysulfide migration towards anode $\rightarrow \mathrm{MgS}_{2}$ film on particles increases

- Formation of $\mathrm{MgS}_{2}$ on anode surface $\rightarrow$ Capacity loss
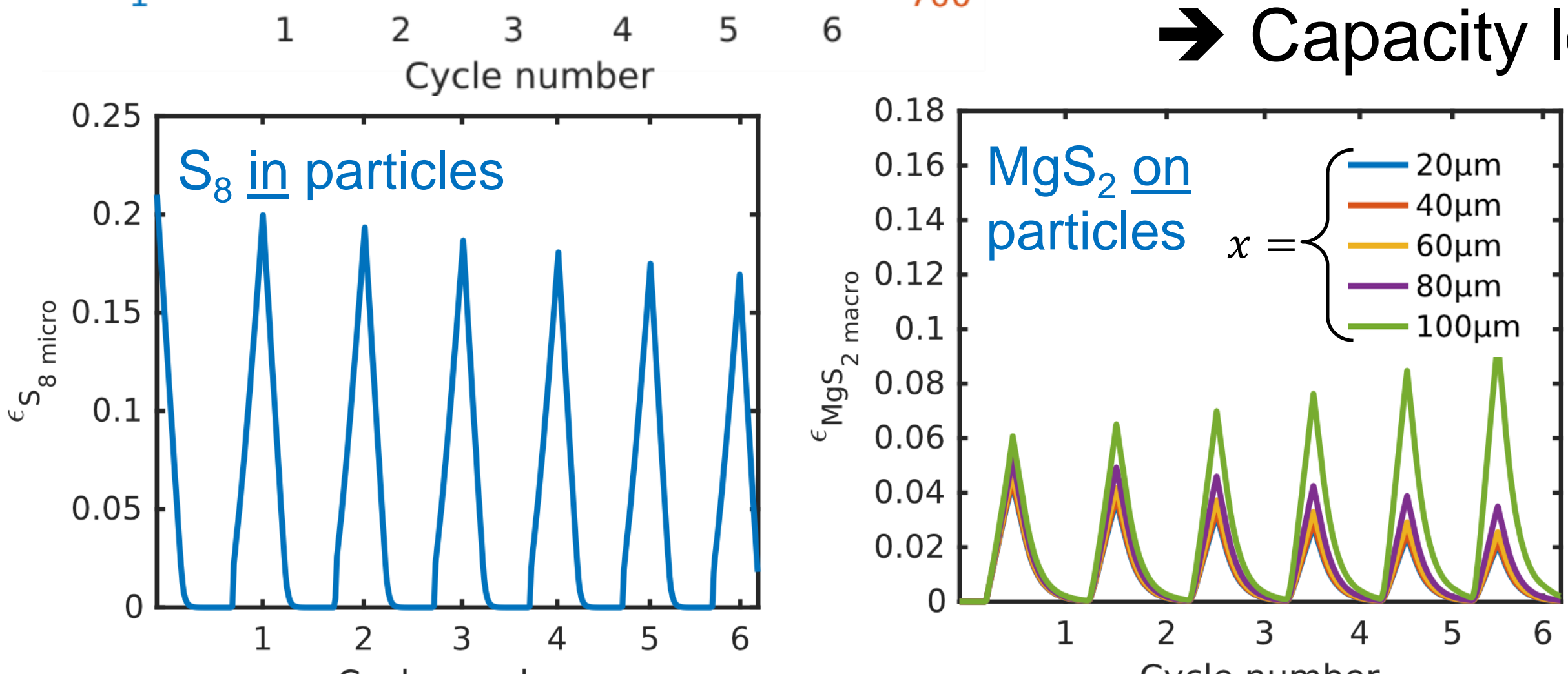

cycle number

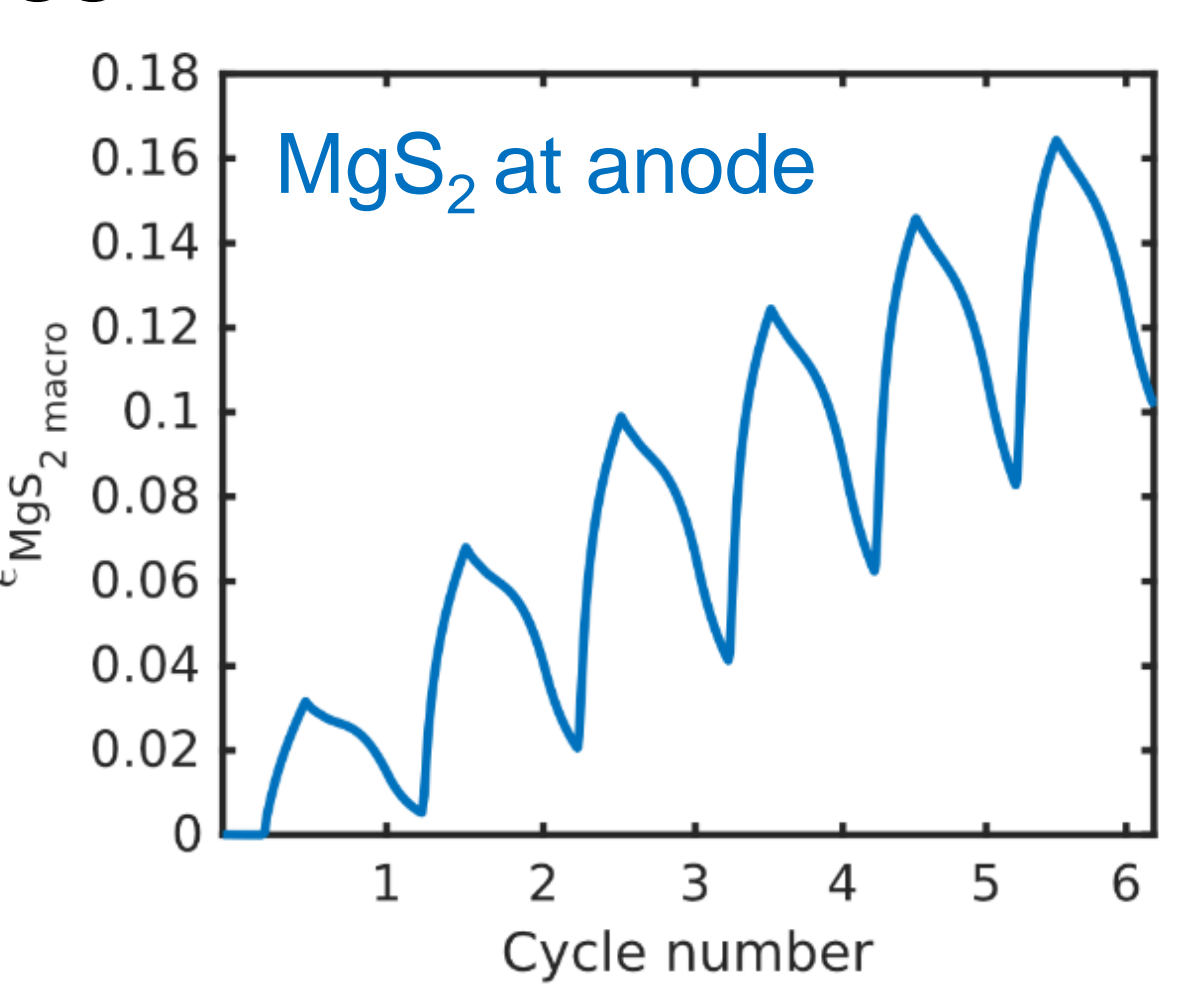

[2] T. Danner et al., Electrochim Acta 184, 124-133 (2015).

[4] Z. Zhao-Karger et al., Adv Energy Mater. 5, 1-9 (2015). 